\title{
MODIFICATION OF DELAYED FEEDBACK CONTROL USING ERGODICITY OF CHAOTIC SYSTEMS
}

\author{
V. Pyragas and K. Pyragas \\ Semiconductor Physics Institute, Center for Physical Sciences and Technology, A. Goštauto 11, LT-01108 Vilnius, Lithuania \\ E-mail: viktpy@pfi.lt, pyragas@kes0.pfi.lt
}

Received 17 March 2010; revised 8 June 2010; accepted 16 September 2010

\begin{abstract}
We devise a modified delayed feedback control algorithm that allows one to stabilize unstable target states of chaotic systems for any initial conditions placed on a strange attractor. The algorithm is based on ergodicity of chaotic systems. We first let the chaotic system to evolve unperturbed until it approaches the neighbourhood of the target state. Then we activate the controller that stabilizes that target state. We propose a special algorithm that evaluates the closeness of the current state of the system to the target state. For continuous-time systems, this algorithm can be implemented by simple low-pass filters. We demonstrate the efficacy of our algorithm with numerical computations of statistics of successful stabilizations.
\end{abstract}

Keywords: chaos, dynamical systems, delayed feedback control, ergodicity

PACS: 05.45.Gg, 02.30.Yy, 02.30.Ks

\section{Introduction}

The field of controlling the chaos, as it has been introduced by the pioneering work of the Maryland group [1], has developed during the last two decades into one of the most active fields in applied nonlinear science. Although control theory is a well-developed discipline in applied mathematics and engineering science, some new aspects have been emphasized in the physics literature, like noninvasive control for stabilizing the vast number of unstable periodic orbits (UPOs) embedded in a chaotic attractor. An extremely simple control scheme, delayed feedback control (DFC), has been proposed in 1992 [2], which allows the stabilization of time-periodic states when no a priori information about the internal dynamics of the system is available. Nowadays the DFC has become one of the most popular methods in chaos control research [3]. The DFC algorithm is reference-free and makes use of a control signal obtained from the difference between the current state of the system and the state of the system delayed by one period of a target orbit. The method allows a noninvasive stabilization of UPOs in the sense that the control force vanishes when the target state is reached. The controlled system can be treated as a black box, since the method does not require any exact knowledge of either the form of the periodic orbit or the system's equations. Successful implementations of the method in- clude quite diverse experimental systems from different fields of science. For the details of experimental implementations as well as various modifications of the DFC algorithm we refer to the recent review paper [ 4 ].

Although the experimental implementation of DFC is almost trivial, the price one has to pay from the theoretical point of view is that the discussion of DFC schemes requires the analysis of differential-difference equations. Such systems are quite difficult to handle since the dynamics takes place in infinite-dimensional phase spaces. The standard tool for discussing the control performance consists in linear stability analysis [5-16]. As a particular benefit of such concepts one has now gained a quite complete overview of the control performance of DFC from the local point of view. But even if such a local analysis predicts stable states, experimental success is not guaranteed, because the control performance may strongly depend on initial conditions. The analysis of global properties of DFC systems, such as basins of attraction of stabilized orbits, is a much more complicated problem. There exists virtually no systematic investigation of time-delayed feedback control beyond the linear regime.

Numerical analysis of particular systems shows that the DFC algorithm can form incredibly complex basins of attraction. In [17, 18] it has been shown that one should pay attention to the global dynamics besides linear stability in understanding the behaviour of the 
controlled system and designing the control parameters. For instance, it is completely unclear how large the basins of attraction are, i.e. which initial condition is attracted towards a particular stabilized orbit. It has also been noted that some targeting method is additionally needed for avoiding unexpected stabilization of an orbit which has the same period with the target orbit.

Although the general results concerning global properties of DFC systems are missing, recently the first step has been taken in understanding a possible mechanism responsible for the size of basins of attraction in DFC schemes [19-21]. In [20], the authors have developed a theory which states that depending on the type of transition at the control boundary there appear basins of attraction of different size. The above authors have expected that the basin of attraction is large when a continuous transition at the control boundary appears; on the other hand, a discontinuous transition at the control boundary indicates that stabilization works only in some neighbourhood of the target state. However, this approach is not universal and does not guarantee the correct prediction for the system parameters far away from the bifurcation point. The lack of a general theory, concerning the global properties of DFC systems, represents a serious drawback of the method.

To improve the global properties of the DFC algorithm several nonlinear modifications have been proposed. A first heuristic idea has been suggested in the original paper [2]. It has been shown that limiting the size of the control force by a simple cut-off increases the basin of attraction of the stabilized orbit. This idea has proved itself in a number of chaotic systems and now it is widely used in experiments. An alternative two-step DFC algorithm has been considered in Ref. [22]. In the first step this algorithm generates an extraneous stable periodic orbit close to the target orbit and in the second step it stabilizes the target. Finally, a nonlinear DFC for systems close to a subcritical Hopf bifurcation has been proposed in Ref. [21]. Here the basin of attraction is enlarged by coupling control forces through the phase of the signal.

However, the above nonlinear DFC schemes are suitable only for specific systems. In this paper, we are using the ergodicity in order to improve the global properties of DFC. Ergodicity is the universal property of chaotic systems. This feature means that the chaotic trajectory visits the close neighbourhood of any orbit with finite probability. In the seminal paper by Ott, Grebogi, and Yorke (OGY) [1], the idea to use the ergodicity in chaos control was formulated for the first time. However, a straightforward implementation of ergodicity in
DFC schemes has not been considered so far. Our aim is to fill this gap and use the OGY ideas for DFC algorithm. Note that unlike the OGY algorithm the perturbation in the DFC algorithm increases the phase dimension of the closed-loop system.

In this paper, we consider two discrete-time systems under DFC, namely, the logistic map in Sec. 2 and the Hénon map in Sec. 3. In these two problems, we let the system to evolve unperturbed until it approaches a close neighbourhood of the target steady state. At this moment we activate the DFC perturbation that stabilizes the target state. The algorithm does not require a knowledge of location of the target. We also consider the stabilization of continuous-time non-autonomous double-well Duffing oscillator in Sec. 4. Note that in Ref. [23] we have already realized the selection and stabilization of one of three period-one UPOs embedded in the chaotic attractor. However, here we consider the selection and stabilization of a period-two orbit. This problem is much more complicated since it requires the use of an extended DFC (EDFC) algorithm [5], and the knowledge of the time average of the displacement of the target orbit. We first let the chaotic system to evolve unperturbed until it approaches the close neighbourhood of the target orbit. As soon it happens, we activate the EDFC feedback perturbation that contains a Gaussian multiplier. This multiplier automatically zeroes the perturbation as long as the solution moves far away from the target. As soon as the target is approached, the feedback perturbation begins to act until it stabilizes the target or moves away from it. The approach to and remoteness from the target state can be repeated several times until the final stabilization of the target is achieved. We finish the paper with conclusions presented in Sec. 5 .

\section{Controlling the logistic map}

Time-discrete maps are very convenient dynamical toy models for analysis of the DFC algorithm. Such systems are easer to handle since the dimension of phase space stays finite even if the control loop is included. The trends discovered through analysis of discrete maps are a good starting point for developing intuition about the behaviour of continuous systems. Moreover, in systems with slow dynamics, the schemes for controlling discrete maps may be directly implemented.

We start our analysis with the simple 1D logistic map $x_{n+1}=b x_{n}\left(1-x_{n}\right)$. For $b>3$ the system has two unstable fixed points, $\tilde{x}_{\mathrm{F}}=0$ and $x_{\mathrm{F}}=1-1 / b$. Our aim is to stabilize the nonzero fixed point $x_{\mathrm{F}}$ by using 
the DFC algorithm $x_{n+1}=b x_{n}\left(1-x_{n}\right)+k\left(x_{n}-\right.$ $\left.x_{n-1}\right)$, where $k$ is the feedback gain. Introducing an auxiliary variable $y_{n}=x_{n-1}$ the latter equation can be rewritten in the form of 2D map:

$$
\begin{aligned}
& x_{n+1}=b x_{n}\left(1-x_{n}\right)+k\left(x_{n}-y_{n}\right), \\
& y_{n+1}=x_{n} .
\end{aligned}
$$

The fixed points of this map are $\left(\tilde{x}_{\mathrm{F}}, \tilde{y}_{\mathrm{F}}\right)=(0,0)$ and $\left(x_{F}, y_{F}\right)=(1-1 / b, 1-1 / b)$. From linear analysis of Eqs. (1) it follows that the nonzero fixed point becomes stable for the values of the feedback gain in the interval

$$
\frac{b-3}{2}<k<1 \text {. }
$$

The optimal value of the feedback gain, which leads to the fastest convergence of nearby initial conditions towards the desired fixed point, is given by

$$
k_{\mathrm{op}}=b-2(b-1)^{1 / 2} .
$$

In order to obtain this result we have to linearize the system (1) around the target fixed point $\left(x_{\mathrm{F}}, y_{\mathrm{F}}\right)$. The linearization yields

$$
\left(\begin{array}{l}
\delta x_{n+1} \\
\delta y_{n+1}
\end{array}\right)=\left(\begin{array}{cc}
2-b+k-k \\
1 & 0
\end{array}\right)\left(\begin{array}{l}
\delta x_{n} \\
\delta y_{n}
\end{array}\right)
$$

Here $\delta x_{n}=x_{n}-x_{\mathrm{F}}$ and $\delta y_{n}=y_{n}-y_{\mathrm{F}}$ are the deviations from target fixed point. The eigenvalues of the Jacobian are given by

$$
\lambda_{1,2}=\frac{\sigma \pm\left(\sigma^{2}-4 \Delta\right)^{1 / 2}}{2},
$$

where $\sigma=2-b+k$ and $\Delta=k$ are the trace and determinant of the Jacobian, respectively. The feedback gain $k$ is optimal when the magnitude of leading eigenvalue is minimal. Such a situation occurs if the discriminant in (5) is zero, i.e. $\sigma^{2}=4 \Delta$,

$$
(2-b+k)^{2}=4 k \text {. }
$$

Solving this equation in respect to $k$, we get

$$
k_{ \pm}=b \pm 2(b-1)^{1 / 2} .
$$

The root $k_{-}$corresponds to the case for which the magnitudes of $\left|\lambda_{1,2}\right|$ are minimal, and thus $k_{\mathrm{op}}=k_{-}$as stated in (3).

In the following we fix the value of the parameter $b=4$, which corresponds to a chaotic regime of the free $(k=0)$ logistic map. Then the optimal gain is $k_{\mathrm{op}} \approx$ 0.536 .

Although the logistic map represents a one-dimensional system, the DFC force increases its dimension to

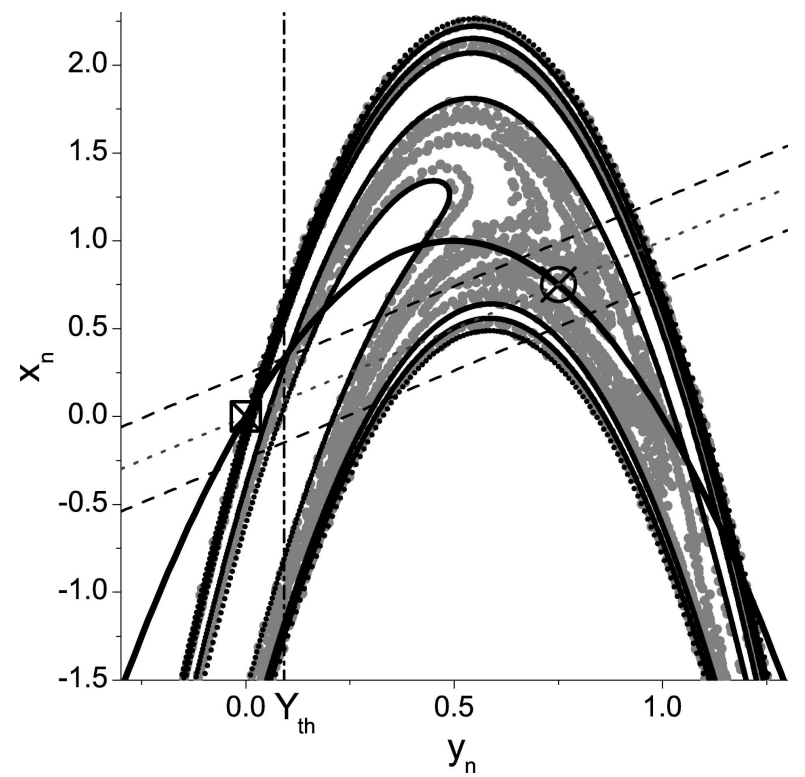

Fig. 1. The phase plane of DFC controlled logistic map (1). Crossed circle and square indicate the target $\left(x_{\mathrm{F}}, y_{\mathrm{F}}\right)$ and zero $\left(\tilde{x}_{\mathrm{F}}, \tilde{y}_{\mathrm{F}}\right)$ fixed points, respectively. The bold curve is the parabola $x_{n}=b y_{n}(1-$ $\left.y_{n}\right)$ representing an attractor of the free logistic map. The dotted line is the identity line $x_{n}=y_{n}$ and two straight dashed lines $x_{n}^{ \pm}=y_{n} \pm \varepsilon$ parallel to the identity line indicate the boundaries of condition 11a). The vertical line $y_{n}=Y_{\text {th }}$ shows the boundary of condition (11b). The thin black dots show the stable manifold of the zero fixed point (in some regions they are blurred to a black line). The large grey dots show the domain of attraction of the target fixed point $\left(x_{\mathrm{F}}, y_{\mathrm{F}}\right)$. The values of parameters are $b=4.0, k=k_{\mathrm{op}} \approx$ $0.536, \varepsilon=0.24$.

two. If we considered a stabilization of a period-two cycle of the logistic map the dimension of the controlled system would be three. The main difference between the DFC and OGY algorithms is that the first increases the dimension of the system under control while the second preservers it unchanged. Note that the phase space dimension of continuous-time systems under DFC control increases to infinity, while for time-discrete systems it remains finite.

Here we restrict ourselves by consideration of a simple fixed point of the logistic map, since we can visualize the closed-loop system dynamics (1) on a 2D plane. The phase portrait of the system is depicted in Fig. 1 . The basin of attraction of the target fixed point $\left(x_{\mathrm{F}}, y_{\mathrm{F}}\right)$ is shown by grey dots. It has been determined by iterating the inverse map

$$
\begin{aligned}
& x_{n-1}=y_{n}, \\
& y_{n-1}=y_{n}+\frac{-x_{n}+b y_{n}\left(1-y_{n}\right)}{k} .
\end{aligned}
$$

of Eqs. (1) starting from many initial conditions located in a small circle around the fixed point $\left(x_{\mathrm{F}}, y_{\mathrm{F}}\right)$. 
We see that the basin of attraction of the target point $\left(x_{\mathrm{F}}, y_{\mathrm{F}}\right)$ is very complex. This complexity can be partially understood by analysis of invariant manifolds of the zero fixed point $\left(\tilde{x}_{\mathrm{F}}, \tilde{y}_{\mathrm{F}}\right)$. The zero fixed point is a saddle and its stable manifold appears to be a boundary curve that confines the basin of attraction of the target fixed point. The stable manifold of the zero fixed point (thin black dots in Fig. 1) was depicted as follows. For the map (1) linearized at the zero fixed point $\left(\tilde{x}_{\mathrm{F}}, \tilde{y}_{\mathrm{F}}\right)$, we first defined the eigenvector

$$
\phi_{s}=\left[1, \frac{b+k}{\lambda_{s}^{2}+k}\right]
$$

of the stable eigenvalue

$$
\lambda_{\mathrm{s}}=\frac{b+k-\left[(b+k)^{2}-4 k\right]^{1 / 2}}{2} .
$$

Then the stable manifold of the original map (1) was plotted by iterating the inverse map (8) from initial conditions placed close to the zero fixed point in the direction of eigenvector (9). In Fig. 1 we see that the stable manifold of the zero fixed point confines the grey dots, i. e. it indeed defines the boundaries of attraction of the target fixed point. Thus the complexity of the basin of attraction of the target fixed point is related with tangled structure of the stable manifold of the zero fixed point.

Although the whole basin of attraction is very complex, it necessarily occupies some region in the vicinity of the target point $\left(x_{\mathrm{F}}, y_{\mathrm{F}}\right)$. This general feature of the basin of attraction results from linear stability of the fixed point. Thus the DFC algorithm should be successful if the initial conditions are in fair proximity to the target. On the other hand, due to the ergodicity, the free system $(k=0)$ should approach the target fixed point as close as desired for any initial conditions placed on the strange attractor.

Taking into account the above facts we propose the following strategy based on DFC, which guarantees successful stabilization of the desired fixed point for any initial conditions. First we set $k=0$, and begin iterations of the free logistic map (1). The points fall on the parabola $x_{n}=b y_{n}\left(1-y_{n}\right)$ shown in Fig. 1 by bold curve. At every step of iterations we check two conditions:

$$
\begin{array}{r}
\left|x_{n}-y_{n}\right|<\varepsilon, \\
y_{n}>Y_{\text {th }},
\end{array}
$$

with $Y_{\text {th }}$ being a threshold value. As soon as both conditions are satisfied we switch on the DFC force with the parameter $k$ chosen in the interval of stability (2) and stabilize the fixed point. In the following we choose the optimal value of the control gain $k=k_{\mathrm{op}}$.

By first condition (11a) we estimate the strength of DFC perturbation if it would be applied at the given iteration step $n$. In Fig. 1 this condition represents a region between two thin straight lines $x_{n}^{ \pm}=y_{n} \pm \varepsilon$. The parameter $\varepsilon$ defines the distance between these lines. For suitably small $\varepsilon$, the lines separate a fairly small segment of the parabola $x_{n}=b y_{n}\left(1-y_{n}\right)$ in the vicinity of the target such that this segment becomes enclosed in the basin of attraction of the target point. Note that the above lines separate two segments of the parabola one close to the desired fixed point and another close to the zero fixed point. The second condition $(11 \mathrm{~b})$ is necessary to exclude the segment in the vicinity of the zero fixed point. The threshold value $Y_{\text {th }}$ is defined as intersection of the parabola $x_{n}=b y_{n}\left(1-y_{n}\right)$ with the upper line $x_{n}^{+}=y_{n}+\varepsilon$ and is given by

$$
Y_{\mathrm{th}}=\frac{b-1-\left[(1-b)^{2}-4 b \varepsilon\right]^{1 / 2}}{2 b} .
$$

By second condition $(11 \mathrm{~b})$ we avoid an activation of the control force in the false region close to the zero fixed point. This suggests that in general a detailed knowledge of the considered system is necessary.

In Fig. 2 we demonstrate the performance of our algorithm for different values of the parameter $\varepsilon$. We show the distribution of times $N$ (number of iterations) needed to achieve control from different initial conditions $y_{0}$. We suppose that $x_{0}=b y_{0}\left(1-y_{0}\right)$, i.e. the initial conditions of the map (1) are placed on the strange attractor of the free system. For a given initial condition $\left(x_{0}, y_{0}\right)$, the total time $N$ consists of two time-spans, $N=N_{1}+N_{2}$. The first time $N_{1}$ represents a number of iterations of the free system needed to approach the neighbourhood of the target point defined by conditions (11). This time describes a transient behaviour of the free system and is determined by the ergodic properties of the chaotic attractor [1]. The second term $\mathrm{N}_{2}$ represents a time of stabilization, i. e. the number of iterations of controlled system needed to approach the stabilized fixed point with a given accuracy $\varepsilon_{\mathrm{F}} \ll \varepsilon$. We assume that control is successful if $\left[\left(x_{N}-x_{\mathrm{F}}\right)^{2}+\left(y_{N}-y_{\mathrm{F}}\right)^{2}\right]^{1 / 2}<\varepsilon_{\mathrm{F}}$. Here we choose $\varepsilon_{\mathrm{F}}=5 \cdot 10^{-3}$. The time $N_{2}$ is defined by the eigenvalues of the stabilized fixed point.

In Fig. 2(a) the parameter $\varepsilon=0.4$ is too large to guarantee the success of the algorithm for any initial conditions. Only $78.9 \%$ of initial conditions taken from the unity interval $y_{0} \in(0,1)$ are successful. The mean time of successful control is $\langle N\rangle \approx 19.64$. In Fig. 2 (b) the 

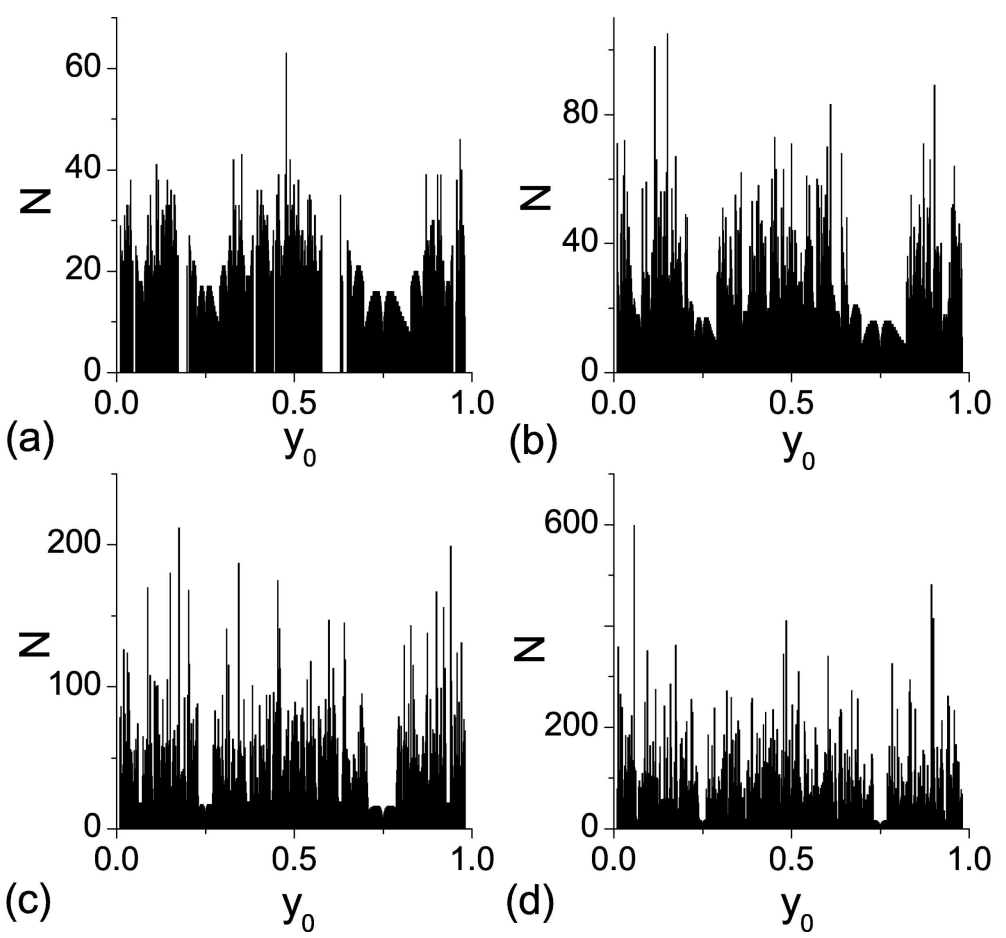

Fig. 2. Distribution of times $N$ needed to achieve control in the logistic map (1). Initial conditions $\left(x_{0}, y_{0}\right)$ are chosen on the attractor of the free system, $x_{0}=b y_{0}\left(1-y_{0}\right)$. The parameter $\varepsilon$ and the mean time $\langle N\rangle$ are (a) $\varepsilon=0.4,\langle N\rangle=19.64$; (b) $\varepsilon=0.24,\langle N\rangle=24.64$; (c) $\varepsilon=$ $0.12,\langle N\rangle=39.60$; (d) $\varepsilon=0.06,\langle N\rangle=71.83$. In (a) only $78.9 \%$ of initial conditions are successful; the empty (white) windows correspond to initial conditions for which the algorithm fails. $\langle N\rangle$ for panel (a) includes only successful realizations. In (b-d) the $100 \%$ success rate is obtained.

parameter $\varepsilon$ is equal to the critical value $\varepsilon_{\mathrm{c}}=0.24$ at which the algorithm produces $100 \%$ success rate for any initial conditions. Now the mean time is $\langle N\rangle \approx 24.64$. With the decrease of the parameter $\varepsilon$ below the critical value, the algorithm also guarantees the successful stabilization for any initial conditions, however, the mean time increases drastically. For $\varepsilon$ equal to 0.12 and 0.06 the mean time $\langle N\rangle$ is respectively 39.60 and 71.83 (Fig. 2(c,d)). Thus the fastest and still reliable convergence of the system towards the desired fixed point is attained for $\varepsilon=\varepsilon_{\mathrm{c}}$.

Why is the success rate not $100 \%$ for larger values of $\varepsilon>\varepsilon_{\mathrm{c}}$ ? The reason for this is that the term of control perturbation disturbs the original system, and for some initial conditions the solution escapes to infinity. The larger $\varepsilon$ makes the boundaries in Fig. 1 wider. Unfortunately, one cannot see the reason for the failure from this figure, and the critical value $\varepsilon_{\mathrm{c}}$ can be found only numerically by iterating the controlled map.

\section{Controlling the Hénon map}

Now we apply our algorithm to a more complex discrete-time chaotic system. We consider the Hénon map [24] subjected to DFC:

$$
\begin{aligned}
& x_{n+1}=1-a x_{n}^{2}+b y_{n}+k\left(x_{n}-y_{n}\right), \\
& y_{n+1}=x_{n} .
\end{aligned}
$$

The Hénon map is the 2D dynamical system described by two variables $\left(x_{n}, y_{n}\right)$. In the following we fix the values of the parameters of the Hénon map at $a=1.5$ and $b=0.2$. The last term in Eq. (13a) describes the DFC force, where $k$ is the feedback gain. Generally, the DFC perturbation should increase the dimensionality of the map, but here it is not the case. The exception here is related to the specific form of the second equation of the Hénon map, $y_{n+1}=x_{n}$. In fact it represents the definition of the time-delayed observable $x_{n-1}=y_{n}$ employed in the DFC force. This feature of the Hénon map allows us to visualize its dynamics on the $2 \mathrm{D}$ plane even in the presence of the control force.

The free as well as controlled Hénon map possesses 


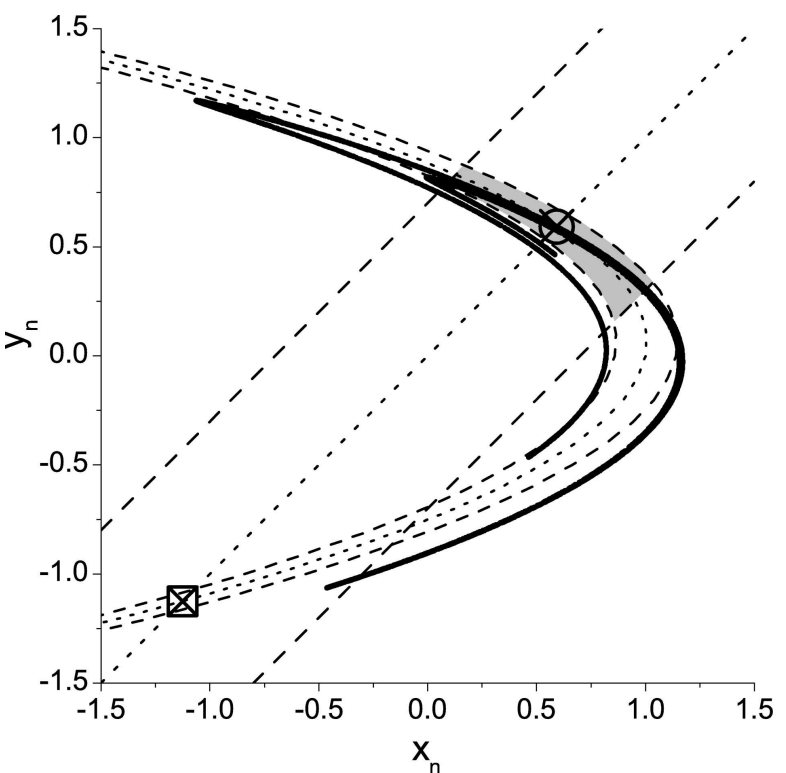

Fig. 3. The phase portrait of the free Hénon map (13) for $a=1.5$ and $b=0.2$. The black dots show the strange attractor. The crossed circle and square denote the target $\left(x_{\mathrm{F}}, y_{\mathrm{F}}\right)$ and extraneous $\left(\tilde{x}_{\mathrm{F}}, \tilde{y}_{\mathrm{F}}\right)$ fixed points, respectively. The straight dash lines $x_{n}^{ \pm}=y_{n} \pm \varepsilon$ define the boundaries of inequality (17a). The inverse transformation of these lines shown by dashed parabolas $x_{n}^{ \pm}=1+b y_{n}-a y_{n}^{2} \pm \varepsilon b$ define the boundaries of inequality $(17 \mathrm{~b})$. The whole region restricted by conditions $(17)$ is marked by grey colour. The identity line $x_{n}=y_{n}$ and its inverse transformation are shown by dotted lines. Their intersections produce the fixed points. The boundary lines are depicted for $\varepsilon=0.7$.

two fixed points, $\left(x_{\mathrm{F}}, y_{\mathrm{F}}\right)=\left(x_{1}^{*}, x_{1}^{*}\right)$ and $\left(\tilde{x}_{\mathrm{F}}, \tilde{y}_{\mathrm{F}}\right)=$ $\left(x_{2}^{*}, x_{2}^{*}\right)$, where

$$
x_{1,2}^{*}=\frac{b-1 \pm\left[(1-b)^{2}+4 a\right]^{1 / 2}}{2 a} .
$$

In Fig. 3 we show the phase portrait of the free $(k=0)$ Hénon map. We see that the fixed point $\left(x_{\mathrm{F}}, y_{\mathrm{F}}\right)$ is embedded in the chaotic attractor, while the point $\left(\tilde{x}_{\mathrm{F}}, \tilde{y}_{\mathrm{F}}\right)$ is an extraneous fixed point, which is outside of the attractor. Our aim is to devise the DFC algorithm, which is able to stabilize the fixed point $\left(x_{\mathrm{F}}, y_{\mathrm{F}}\right)$ for any initial conditions placed on the strange attractor.

From linear analysis of the map (13) it follows that the target fixed point $\left(x_{\mathrm{F}}, y_{\mathrm{F}}\right)$ is stable for values of the feedback gain in the interval

$$
\left(b-1+2 a x_{1}^{*}\right) / 2<k<b+1 .
$$

An optimal value of the feedback gain providing the fastest convergence to the target state is given by

$$
k_{\mathrm{op}}=2\left[a x_{1}^{*}+1-\left(2 a x_{1}^{*}+1-b\right)^{1 / 2}\right] .
$$

For the chosen values of parameters we get $k_{\mathrm{op}} \approx 0.566$. In the following we use this value in our control algorithm.
The linear stability guarantees that the basin of attraction of the target point for the nonlinear map (13) occupies some region around this point. As well as in the previous section, we need conditions which allow us to check whether the current state of the free system is in vicinity of the target state. For 2D map we require two conditions in order to separate some region around the target point. Our aim is to formulate these conditions without any knowledge of even approximate position of the fixed point. Moreover, we suppose that only one scalar variable, say $x_{n}$, is available for observation. Then we formulate the desired conditions as follows: $\left|x_{n}-x_{n-1}\right|<\varepsilon$ and $\left|x_{n-1}-x_{n-2}\right|<\varepsilon$. This means that we check the smallness of DFC perturbation if it would be applied not only at the current moment $n$ but also at the previous time $n-1$. Since $x_{n-1}=y_{n}$, we can rewrite the above conditions as follows:

$$
\begin{array}{r}
\left|x_{n}-y_{n}\right|<\varepsilon, \\
\left|x_{n-1}-y_{n-1}\right|<\varepsilon .
\end{array}
$$

For an $M$-dimensional map we would write $M$ analogous conditions.

Geometrically, conditions (17) separate some regions in the $\left(x_{n}, y_{n}\right)$ plane in the vicinity of the both fixed points. In Fig. 3, the region surrounding the target point is marked by grey colour. The region is bounded by four curves. The inequality (17a) defines the region between two straight lines $x_{n}^{ \pm}=y_{n} \pm \varepsilon$ parallel to the identity line. The inequality (17b) bounds the region between two parabolas $x_{n}^{ \pm}=1+b y_{n}-a y_{n}^{2} \pm \varepsilon b$, which represent an inverse Hénon transformation of the above lines. With the decrease of parameter $\varepsilon$, the region defined by conditions (17) shrinks and for suitably small $\varepsilon$ should fit into the basin of attraction of the target point. Note that for the Hénon system we do not need an additional condition like (11b) to exclude the extraneous fixed point $\left(\tilde{x}_{\mathrm{F}}, \tilde{y}_{\mathrm{F}}\right)$ since here this fixed point is outside of the strange attractor.

In Fig. 4 we demonstrate the performance of our algorithm for different values of the parameter $\varepsilon$. To gather statistics on times needed to achieve control we apply our algorithm for many different initial conditions placed on the strange attractor of the free Hénon map. For a given initial condition $\left(x_{0}, y_{0}\right)$ the procedure is as follows. First, we set $k=0$ and start an iteration of the free map (13). On each step of iteration we check the conditions (17). As soon as these conditions are satisfied we switch on the control force by setting $k=k_{\mathrm{op}}$. Then we continue the iterations until the system approaches the desired fixed point with a given accuracy 

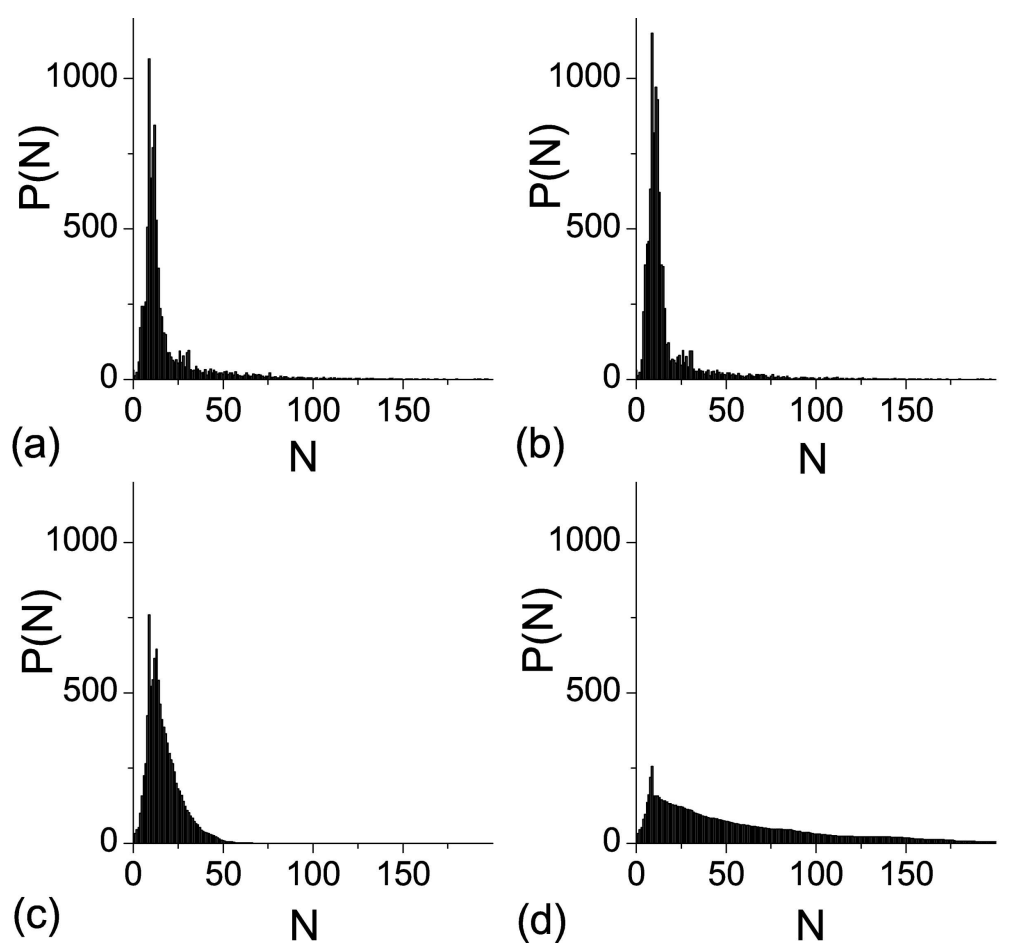

Fig. 4. Histogram of time $N$ needed to achieve control in the Hénon map 113$)$. $P(N)$ shows the number of successful stabilizations with the given time of control. The parameter $\varepsilon$ and the mean time $\langle N\rangle$ are (a) $\varepsilon=2.7,\langle N\rangle=19.44$; (b) $\varepsilon=1.77,\langle N\rangle=17.41$; (c) $\varepsilon=0.7,\langle N\rangle=$ 16.93; (d) $\varepsilon=0.2,\langle N\rangle=59.27$. In (a) only $86.92 \%$ of initial conditions are successful, while in (b-d) the $100 \%$ success rate is obtained.

$\varepsilon_{\mathrm{F}}=2 \cdot 10^{-3} \ll \varepsilon$. As this happens we record the total time $N$ (number of iterations) elapsed from the start to the end of this procedure. We repeat this procedure for $10^{4}$ different initial conditions randomly chosen on the strange attractor and plot a histogram of time $N$ needed to achieve the control. If the value of the parameter $\varepsilon$ is too large, then the algorithm fails for some part of initial conditions (Fig. 4(a)). By decreasing the parameter $\varepsilon$ we can gain $100 \%$ success rate Fig. $4(b-d)$, similarly as for the logistic map. However, unduly small $\varepsilon$ can lead to rather long mean time $\langle N\rangle$.

We have included in Fig. 3 the grey region only for the value of $\varepsilon$ used in Fig. 4 (c) that serves as an illustrative example. The grey region for other cases of Fig. 4 would seem similarly to the shown one.

Exactly as in the case of logistic map (Sec. 2), the success rate is not $100 \%$ for larger values of $\varepsilon>\varepsilon_{\mathrm{c}}$. The reason for this is the same as described in Sec. 2 .

\section{Controlling the Duffing oscillator}

In the two previous sections, we analysed the discrete systems, namely, the logistic map and the Hénon map controlled by modified DFC algorithm. Here, however, we are going to consider the continuous system, namely, the EDFC controlled non-autonomous doublewell Duffing oscillator:

$$
\begin{aligned}
& \dot{x}=y-G(t), \\
& \dot{y}=-\beta y+\alpha x-\gamma x^{3}+A \cos (\Omega t) .
\end{aligned}
$$

Here $x, y$ are the dynamic variables, $\alpha=1, \gamma=1$ are the parameters of the double-well potential, $\beta=0.16$ is the coefficient of losses, $A=0.27$ and $\Omega=1$ are the amplitude and frequency of the external force, respectively.

We assume that $x(t)$ can be measured and one can add a perturbation $G(t)$ at the r.h.s. of $(18 \mathrm{a})$. This perturbation is constructed using the delayed difference

$$
D(t)=x(t)-x(t-\tau) .
$$

The delay $\tau$ must coincide with the period of the target orbit. For the chosen parameters, the free system (18) $(G=0)$ exhibits the chaotic motion.

The chaotic attractor contains three period-one orbits (with period $\tau=2 \pi / \Omega=2 \pi$ ). One of them (in the origin) satisfies the odd-number limitation [14, 15], and its stabilization with an unstable controller [16] has been considered in the recent paper [22]. The other period-one orbits are located on the left and the right 


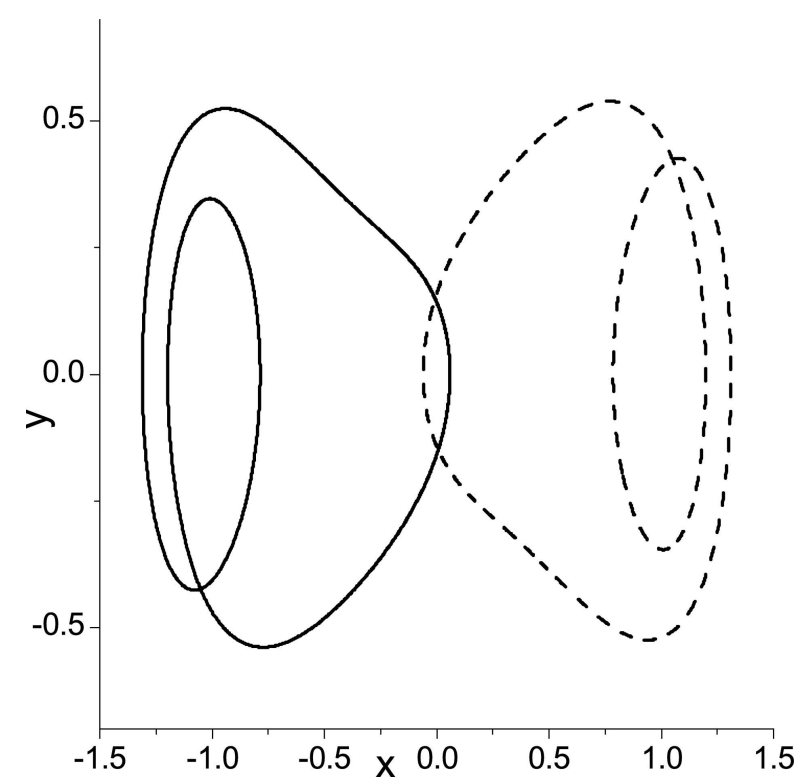

Fig. 5. Two period-two partner UPOs embedded in chaotic attractor of the free $(G=0)$ double-well oscillator 18$)$ for $\alpha=1, \beta=0.16$, $\gamma=1, A=0.27$, and $\Omega=1$. The both orbits have the same period

$\tau=4 \pi$. The target (left-hand) orbit is depicted by solid line.

sides in respect to the $y$ axis. We considered the problem of their selection and stabilization in [23]. We used the DFC controller supplemented by two low pass filters that enabled us to select the desired (say, the righthand) orbit, and to estimate the closeness of the solution to the target. Note that this problem was relatively simple since the both UPOs were placed on the different sides of the $y$ axis. In addition, these orbits did not need the extended version of the DFC (EDFC), i.e. it was enough to use the DFC scheme. In the present paper, however, we are considering the stabilization of the period-two UPOs. Their period is $\tau=2 \cdot 2 \pi / \Omega=4 \pi$. Note that there are three different pairs of period-two UPOs embedded in the strange attractor. Here we consider the stabilization of one pair that is shown in Fig. 5 . These two UPOs are crossing the $y$ axis, and the lefthand UPO spends the most time in the region $x<0$, while the right-hand UPO spends in the region $x>0$.

We now make some remarks about their stabilization. First, these UPOs are strongly unstable and their stabilization requires the use of the extended DFC (EDFC) method [5]. Second, if we activate the control perturbation only once (as soon as the solution approaches the close neighbourhood of the target), we will see that some of the random initial conditions lead to the stabilization of desired target, and the other initial conditions to the stabilization of the the other orbit.

In order to achieve the successful stabilization of the desired orbit, we change our control strategy presented in Ref. [23]. It means that we consequently develop the control scheme proposed in Ref. [23] in order to achieve the stabilization of orbits of higher order. First we introduce two low pass filters:

$$
\begin{aligned}
\tau_{w} \dot{w} & =|D(t)|-w, \\
\tau_{v} \dot{v} & =x-v .
\end{aligned}
$$

The first filter (20a) estimates the smallness of the delayed difference. For the $\tau$-periodic solution it should vanish. The second one evaluates the time average of the displacement $x(t)$. If the solution moves around the left-hand orbit, then $v(t)$ oscillates around its average $\left\langle x_{\text {left }}\right\rangle$ with relatively small amplitude. For definiteness we intend to stabilize the left-hand orbit. The stabilization of the right-hand orbit would require to make substitution $\left\langle x_{\text {left }}\right\rangle \rightarrow\left\langle x_{\text {right }}\right\rangle$. The following algorithm enables to distinguish between stabilizations of two period-two partner orbits.

The control procedure is as follows. We analyse the free running system $(G(t)=0)$ by means of the introduced variables $w$ and $v$. We simultaneously check two conditions,

$$
\begin{aligned}
w(t) & <\varepsilon, \\
\left|v(t)-\left\langle x_{\text {left }}\right\rangle\right| & <\varepsilon_{v} .
\end{aligned}
$$

As soon as both conditions are satisfied, we activate the control by setting

$$
G(t)=k_{\mathrm{op}} S(t) \exp \left[-\left(v(t)-\left\langle x_{\mathrm{left}}\right\rangle\right)^{2} / \varepsilon_{v}^{2}\right],
$$

and we do not check the conditions (21) anymore. In (22) $S(t)$ is the feedback perturbation of the EDFC scheme [5]. It can be found from the recurrent relations

$$
\begin{aligned}
& S(t)=x(t)-(1-R) B(t-\tau), \\
& B(t)=x(t)+R B(t-\tau) .
\end{aligned}
$$

We need a single delay line for the auxiliary variable $B(t) . R$ is the parameter of the EDFC. For $R=0$, this scheme reduces to the original DFC method.

Now we discuss the control perturbation (22). In this expression, the usual feedback perturbation $k_{\mathrm{op}} S(t)$ is multiplied by the Gaussian function. Let us suppose that the solution moves far away from the target orbit for a characteristic time interval $\left(t-\tau_{v}, t\right)$. Then we have

$$
\left|v(t)-\left\langle x_{\text {left }}\right\rangle\right| \gg \varepsilon_{v} .
$$

In such a case, the Gaussian exponent in (22) will be almost zero, and $G(t) \simeq 0$. On the other hand, if the 
solution runs around the close neighbourhood of the target orbit for a characteristic time interval $\left(t-\tau_{v}, t\right)$, we get

$$
\left|v(t)-\left\langle x_{\text {left }}\right\rangle\right| \ll \varepsilon_{v} .
$$

This means that in Eqs. (22) the exponent will be almost unity thus yielding $G(t) \simeq k_{\mathrm{op}} S(t)$.

Summing up we conclude that after the activation, the controller acts only in the neighbourhood of the target orbit. When the solution runs away, the control perturbation vanishes until the next approach to the target.

The linear stability analysis (for $R=0.5$ ) shows that the target orbit is stable for $0.54<k<0.72$. The optimal value of the feedback gain is $k_{\mathrm{op}}=0.58$. The average value of displacement for the left-hand orbit is $\left\langle x_{\text {left }}\right\rangle=-0.6352$.

In Fig. 6 we illustrate the success of the above EDFC algorithm by numerical computations. In Fig. 6 (a) the dynamics of the variable $v(t)$ is shown. When the solution is chaotic, it wanders up and down. As soon as the stabilization occurs, it is attracted to $\left\langle x_{\text {left }}\right\rangle$, and oscillates around this value with a relatively low amplitude. In Fig. 6(b) we show the dynamics of the feedback perturbation $G(t)$. When the solution is far from the target orbit, this perturbation is turned off. At about $t \simeq 480 \tau$ the solution approaches the neighbourhood of the left-hand orbit, and the feedback perturbation (22) is activated. In Fig. 6(b) we see that the control perturbation is switched on and off several times (because of the Gaussian exponent) until the stabilization of the target occurs. In Fig. 6(c) we show the dynamics of the displacement $x(t)$. Before the stabilization it is chaotic, and after the stabilization it periodically oscillates around $\left\langle x_{\text {left }}\right\rangle$. In Fig. $\sigma(\mathrm{d})$ we present the phase portrait of the controlled Duffing oscillator for the last period of integration of Eqs. (18). The obtained solution exactly coincides with the left-hand UPO in Fig. 5 .

In Fig. 7 we show the statistics of successful stabilizations for $10^{3}$ initial conditions randomly chosen on the chaotic attractor. We have taken the parameters $\tau_{w}=\tau / 20, \tau_{v}=\tau / 0.35, \varepsilon_{v}=0.3$, and varied the parameter $\varepsilon$. For all the values of $\varepsilon$, the algorithm successfully stabilizes the target orbit for $100 \%$ of initial conditions. The optimal value is $\varepsilon=\varepsilon_{\mathrm{op}}=0.2$ since it leads to the minimal average $\langle N\rangle=143.56$. The lower values of $\varepsilon<\varepsilon_{\text {op }}$ require more time for the free chaotic system to approach this narrow window. On the other hand, the larger values of $\varepsilon>\varepsilon_{\text {op }}$ need also the
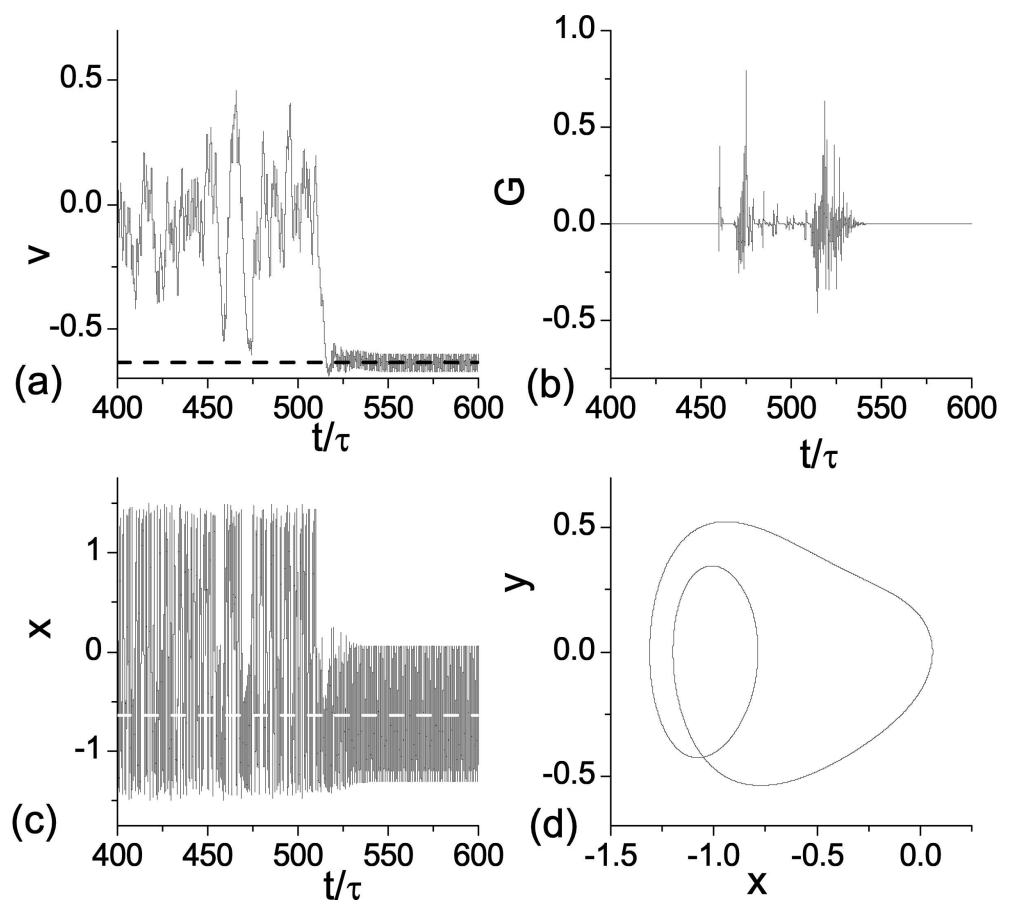

Fig. 6. The results of numerical integration of the EDFC controlled double-well oscillator described by Eqs. (18), (19), (20), (22), and (23). Here it is shown the dynamics of (a) $v(t)$, (b) the feedback perturbation $G(t)$, and (c) the displacement $x(t)$. In (d) there is plotted the phase portrait of the stabilized target orbit (for the last period of integration). The parameters are as follows: $\varepsilon_{v}=0.3, \varepsilon=0.06, \tau_{w}=\tau / 20$, $\tau_{v}=\tau / 0.35, k=k_{\mathrm{op}}=0.58, R=0.5$, and $\tau=4 \pi$. The other parameters are the same as in Fig. 5. The initial conditions are randomly taken on the strange attractor. The dashed horizontal lines in (a) and (c) indicate the time average of the displacement for the target left-hand orbit, $\left\langle x_{\text {left }}\right\rangle=-0.6352$. 

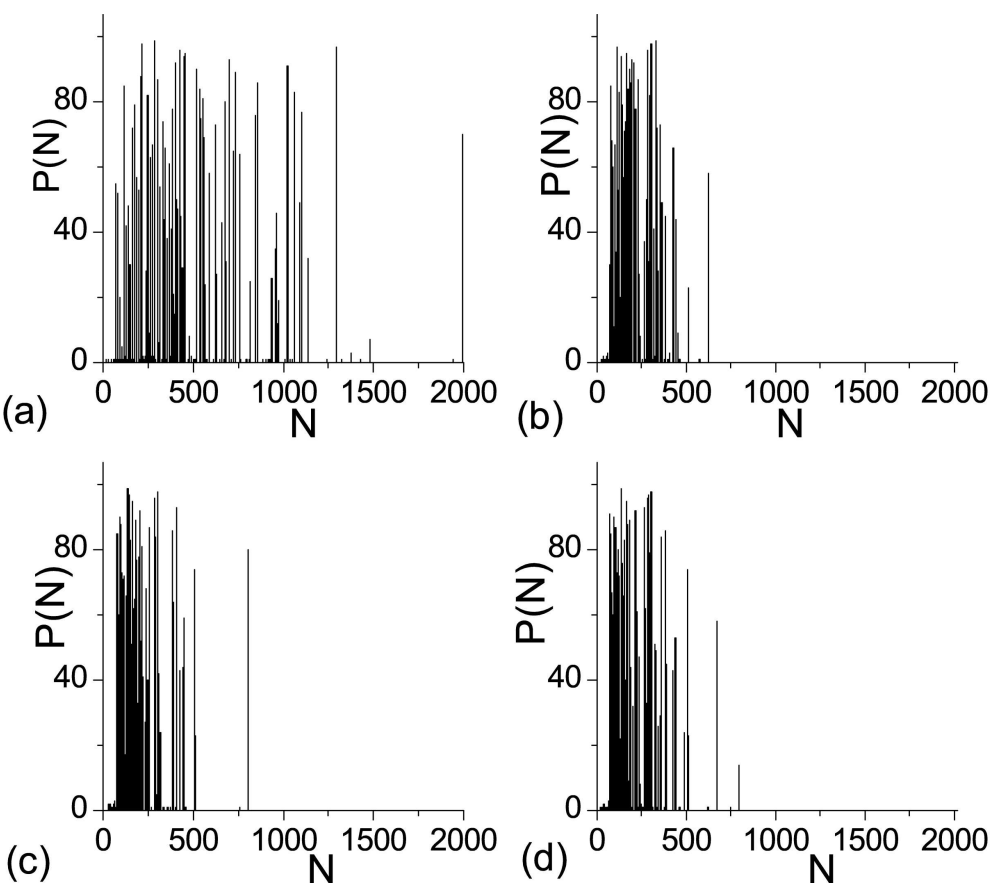

Fig. 7. Histogram of time needed to achieve control in the Duffing system for $k=k_{\mathrm{op}}=0.58, R=0.5, \tau=4 \pi, \tau_{w}=\tau / 20, \tau_{v}=\tau / 0.35$, and $\varepsilon_{v}=0.3 . N$ is the number of periods $\tau$ needed for stabilization of the target UPO and $P(N)$ is the number of successful stabilizations with the given time of control. The parameter $\varepsilon$ and the mean number $\langle N\rangle$ of periods needed to achieve control are (a) $\varepsilon=0.04,\langle N\rangle=$ 465.97; (b) $\varepsilon=0.15,\langle N\rangle=164.42$; (c) $\varepsilon=\varepsilon_{\mathrm{op}}=0.2,\langle N\rangle=143.56$; (d) $\varepsilon=0.25,\langle N\rangle=161.32$. In all the graphs the $100 \%$ success rate is obtained.

longer time of stabilization since the larger values of window cause the disturbances of the system that lead to longer times for controller to find the target orbit. The mean time needed for successful stabilization is approximately equal to 140 periods of UPO for $\varepsilon=\varepsilon_{\mathrm{op}}$. Such a long transient dynamics is related with the small leading Lyapunov exponent $\left(\lambda_{\min }=-0.0348\right)$ of the target orbit for the optimal value of feedback gain $k=k_{\mathrm{op}}$.

The success of control is independent of $\varepsilon$ contrary to the findings on the map models in Sections 2 and 3 , since the methods of turning on the control are different. In Sections 2, 3 we checked the closeness of solution to the target states only once, and as it was satisfied we turned on the control perturbation that continued to act until the success/failure. However, in the case of continuous-time double-well oscillator we also checked the closeness of solution to the target, but here we activated the control perturbation in the form of Gaussian (22); this Gaussian continued to act until the successful end. We stress that the Gaussian perturbation contains the turning on/off in itself, since when the running time average of displacement is near (far away from) the time average of target orbit then the control is on (off). This means that we may activate the Gaussian perturbation (22) at any initial conditions lying on the chaotic attractor. Therefore the conditions (21) are necessary only for faster capture of the target orbit by the Gaussian. We thus conclude that the success of control does not depend on $\varepsilon$.

One may intuitively think that the success of control would depend on the relation of $\varepsilon_{v}$ and the radius of the period-two orbit that is to be stabilized. But apart from these two parameters there is also present the characteristic time $\tau_{v}$, Eq. (20b), of the filter. Therefore we think that the dependence of success on the controller and system parameters is a non-trivial problem.

\section{Conclusions}

We have considered a modified delayed feedback control (DFC) algorithm that provides successful stabilization of unstable periodic orbits (UPOs) of chaotic systems for any initial conditions placed on the chaotic attractor. In our modification we employ the ergodic property of chaotic attractors, which are universal for any chaotic systems. According to this property the chaotic trajectory visits the close neighbourhood of any target state with a finite probability. We wait until the state of the free chaotic system approaches the target orbit, and as soon as this happens, we activate the feedback perturbation that stabilizes this target. 
We have demonstrated the efficiency of our algorithm for two time-discrete systems, namely, the logistic map, and the Hénon map, as well as for the non-autonomous time-continuous double-well Duffing oscillator. With time-discrete systems we were able to visualize geometrically the main ideas of our algorithm. For time-continuous Duffing oscillator, we have introduced two low pass filters. The first of them estimates the delayed difference. The second filter serves to select the desired orbit among several orbits with the same period. Note that in Ref. [23], we have been dealing with selection and stabilization of the period-one orbits embedded in chaotic attractor. Here, however, we solved a much more complex problem, namely, the selection and stabilization of the period-two orbits. Unlike Ref. [23], here we needed to use the extended version of the DFC (EDFC) [5], and we also used a Gaussian multiplier in the EDFC feedback perturbation. The Gaussian multiplier automatically zeroes the perturbation when the solution of the system is far away from the target orbit; the perturbation becomes activated only in the close neighbourhood of the target orbit. One may say that the controller acts by trial and error until the successful stabilization happens.

By choosing many random initial conditions on the chaotic attractor we verified the success of our algorithm statistically. For the both discrete-time systems, we attained $100 \%$ success rate, provided that the windows of closeness of the solution to the target were sufficiently small. In the case of the Duffing oscillator, the reliable stabilization of the desired period-two orbit is attained for any window $\varepsilon$, but there exists an optimal value of window, $\varepsilon=\varepsilon_{\mathrm{op}}$, for which the mean time of stabilization is minimal. The existence of the optimal $\varepsilon_{\mathrm{op}}$ can be explained in the following way. If we decrease the parameter $\varepsilon$, the free system will need more time to approach the close neighbourhood of the target. On the other hand, if we increase the window $\varepsilon$, it may happen that the feedback perturbation will be activated for solutions which are too far from the target, and the perturbation will disturb the original system.

Note that the proposed algorithm is rather simple and can be easily implemented electronically.

\section{Acknowledgement}

We acknowledge the support from the global grant No VP1-3.1-ŠMM-07-K-01-025 "The development of chaos control and synchronization algorithms and their application to complex neural networks".

\section{References}

[1] E. Ott, C. Grebogi, and J.A. Yorke, Controlling chaos, Phys. Rev. Lett. 64, 1196-1199 (1990).

[2] K. Pyragas, Continuous control of chaos by selfcontrolling feedback, Phys. Lett. A 170, 421-428 (1992).

[3] Handbook of Chaos Control, 2nd ed., eds. E. Schöll and H.G. Schuster (Wiley-VCH, Weinheim, 2008).

[4] K. Pyragas, Delayed feedback control of chaos, Philos. Trans. R. Soc. A 364, 2309-2334 (2006).

[5] J.E.S. Socolar, D.W. Sukow, and D.J. Gauthier, Stabilizing unstable periodic orbits in fast dynamical systems, Phys. Rev. E 50, 3245-3248 (1994).

[6] K. Pyragas, Control of chaos via extended delay feedback, Phys. Lett. A 206, 323-330 (1995).

[7] M.E. Bleich and J.E.S. Socolar, Stability of periodic orbits controlled by time-delay feedback, Phys. Lett. A 210, 87-94 (1996).

[8] W. Just, E. Reibold, H. Benner, K. Kacperski, P. Fronczak, and J. Holyst, Limits of time-delayed feedback control, Phys. Lett. A 254, 158-164 (1999).

[9] K. Pyragas, Analytical properties and optimization of time-delayed feedback control, Phys. Rev. E 66, 026207 (2002).

[10] K. Pyragas, V. Pyragas, and H. Benner, Delayed feedback control of dynamical systems at a subcritical Hopf bifurcation, Phys. Rev. E 70, 056222 (2004).

[11] T. Pyragiené and K. Pyragas, Delayed feedback control of forced self-sustained oscillations, Phys. Rev. E 72, 026203 (2005).

[12] V.Pyragas and K. Pyragas, Delayed feedback control of the Lorenz system: An analytical treatment at a subcritical Hopf bifurcation, Phys. Rev. E 73, 036215 (2006).

[13] B. Fiedler, V. Flunkert, M. Georgi, P. Hövel, and E. Schöll, Refuting the odd number limitation of timedelayed feedback control, Phys. Rev. Lett. 98, 114101 (2007).

[14] H. Nakajima, On analytical properties of delayed feedback control of chaos, Phys. Lett. A 232, 207-210 (1997).

[15] W. Just, T. Bernard, M. Ostheimer, E. Reibold, and H. Benner, Limits of time-delayed feedback control, Phys. Rev. Lett. 78, 203-206 (1997).

[16] K. Pyragas, Control of chaos via an unstable delayed feedback controller, Phys. Rev. Lett. 86, 2265-2268 (2001).

[17] K. Yamasue and T. Hikihara, Domain of attraction for stabilized orbits in time delayed feedback controlled Duffing systems, Phys. Rev. E 69, 056209 (2004).

[18] K. Yamasue and T. Hikihara, Persistence of chaos in a time-delayed-feedback controlled Duffing system, Phys. Rev. E 73, 036209 (2006).

[19] C. von Loewenich, H. Benner, and W. Just, Experimental relevance of global properties of time-delayed feedback control, Phys. Rev. Lett. 93, 174101 (2004). 
[20] W. Just, H. Benner, and C. von Loewenich, On global properties of time-delayed feedback control: Weakly nonlinear analysis, Physica D 199, 33-44 (2004).

[21] K. Höhne, H. Shirahama, C.-U. Choe, H. Benner, K. Pyragas, and W. Just, Global properties in an experimental realization of time-delayed feedback control with an unstable control loop, Phys. Rev. Lett. 98, $214102(2007)$

[22] A. Tamaševičius, G. Mykolaitis, V. Pyragas, and K. Pyragas, Delayed feedback control of periodic or- bits without torsion in nonautonomous chaotic systems: Theory and experiment, Phys. Rev. E 76, 026203 (2007).

[23] V. Pyragas and K. Pyragas, Using ergodicity of chaotic systems for improving the global properties of the delayed feedback control method, Phys. Rev. E 80, 067201 (2009).

[24] M. Hénon, A two-dimensional mapping with a strange attractor, Commun. Math. Phys. 50, 69-77 (1976).

\title{
UŽDELSTO GRIZŽTAMOJO RYŠIO VALDYMO MODIFIKACIJA PANAUDOJANT CHAOTINIŲ SISTEMŲ ERGODIŠKUMĄ
}

\author{
V. Pyragas, K. Pyragas \\ Fiziniu ir technologijos mokslu centro Puslaidininkiu fizikos institutas, Vilnius, Lietuva
}

\section{Santrauka}

Pasiūlème modifikuotą uždelsto grižtamojo ryšio valdymo algoritmą, kuris stabilizuoja nestabilias periodines orbitas bei rimties taškus, kai sprendinys startuoja iš bet kurių chaotinio atraktoriaus pradinių sąlygų. Algoritmas yra grindžiamas chaotinių sistemų ergodiškumu. Pirma leidžiame chaotinei sistemai evoliucionuoti lais- vai tol, kol ji priartès prie norimos orbitos. Tuomet ijungiame valdiklį, kuris stabilizuoja norimą orbitą. Pasiūlème algoritmą, kuris ivertina esamo sprendinio artumą norimai būsenai. Tolydžiosioms sistemoms šị algoritmą galima įdiegti paprastais žemų dažnių filtrais. Algoritmo veiksmingumą pademonstravome sékmių statistikos skaitiniais skaičiavimais. 